\title{
Chemical evolution of a classical bulge: The case of M31
}

Monica M. Marcon-Uchida ${ }^{* \dagger}$

Universidade Cruzeiro do Sul (NAT/UNICSUL)

E-mail: monica.uchida@cruzeirodosul.edu.br

\section{Francesca Matteucci}

University of Trieste

E-mail: matteucc@oats.inaf.it

\section{Gustavo A. Lanfranchi}

Universidade Cruzeiro do Sul (NAT/UNICSUL)

E-mail: gustavo.lanfranchiecruzeirodosul.edu.br

The Study of the chemical evolution of nearby galaxies is a very important tool to improve our knowledge about the process of star formation and galaxy evolution. The proximity of Local Group galaxies allows accurate analysis of their chemical properties and stellar populations, that can be used as constraints in theoretical studies. During the last years the structure of bulges has been the subject of intense work. The exisence of classical bulges, pseudo-bulges and their coexistence in the central regions of galaxies are still under discussion. In this work we aim at reproducing the chemical evolution of the bulge of M31 by means of a generalized model in the context of a classical bulge. We compute several models of chemical evolution using the metallicity distribution of dwarf stars as an observational constraint for that stellar system. We compare the results of previous models with our models using standard parameters like the Salpeter initial mass function (IMF) and Schimdt law for the star formation rate (SFR). Using standard parametrization and assuming different timescales for the infall of gas in a classical bulge we reproduce its metallicity distribution of dwarfs and predict the abundance ratios of several elements in the bulge of M31. Our results suggest that the bulge of M31 formed rapidly by means of an intense star formation rate. We can also reproduce the metallicity distribution function using a generalized model with the Salpeter IMF and compare our results with the same model with a flatter IMF. We also predict that the $[\alpha / F e]$ ratios in the stars of the bulge of M31 are high for most of the $[\mathrm{Fe} / \mathrm{H}]$ range.

\section{International Symposium on Nuclei in the Cosmos}

August 5-12, 2012

Cairns, Australia

\footnotetext{
* Speaker.

${ }^{\dagger}$ This research was supported by FAPESP (Proj. 2010/17142-4).
} 


\section{Introduction}

M31 is the largest and more massive galaxy of the Local Group of galaxies. Despite of its vicinity we have a small amount of information to constrain the chemical evolution models, and this is due to its high inclination angle $i=77^{\circ}$ (Walterbros \& Kennicutt 1987) which provide us with an edge-on view of the system. Nevertheless, individual stars were studied in the bulge of this galaxy and the stellar metallicty distribution could be inferred allowing one to investigate its formation.

Bulges are spheroidal stellar systems located in the center of spiral galaxies which usually can be distinguished from the spiral disk by their different dynamics, chemistry and photometric features. Classical bulges present similar properties to elliptical galaxies and are mainly composed by an old stellar population as this type of bulge is supposed to be formed very fast at the beginning of the galactic evolution. Elmegreen (1999) suggested that in a classical bulge the potential well is too deep to have allowed a self-regulation mechanism or gas outflows as it occurs in spiral disks and dwarf galaxies. Therefore, classical bulges should have passed through a very intense star formation, at a maximum rate, during a short timescale.

The bulge of M31 is a clear example of classical bulge (Kormendy \& Kennicutt 2004) and is one of the few bulges for which we could detect individual stars and objects (Sarajedini \& Jablonska 2005, Worthey et al. 2005) that can constrain chemical evolution models.

Matteucci \& Brocato (1990), hereafter MB90, first suggested that to reproduce the evolution of the Milky Way bulge one should assume a fast gas collapse (timescales shorter than $1 \mathrm{Gyr}$ ), a very efficient star formation rate (SFR) and an initial mass function (IMF) flatter than the Salpeter (1955) one. In MB90 they also predicted that the $[\alpha / \mathrm{Fe}]$ should be supersolar over almost the whole metallicity range as a consequence of assuming a fast bulge evolution, and this was later on confirmed by observations (McWilliam \& Rich, 1994).

More recently, Ballero et al. (2007a, BMOR2007) computed a chemical evolution model for the bulge of the Milky Way, confirming that a short star formation rate and a high efficiency are needed to fit the observational constrains avaiable for the Galactic bulge. In this paper, we compute the chemical evolution of the bulge of M31 (classified as a pure classic bulge, Kormedy \& Kennicutt 2004) using a single-zone model as general as possible to compare the chemical evolution of a classical bulge with the previous results obtained based on a model parameterized by the MW bulge (Ballero et al. 2007b, hereafter BKM2007).

\section{Model Prescriptions}

To reproduce the chemical evolution of the M31 bulge we used a generalized version of the model proposed in BKM2007. The prescriptions may be summarized as follows:

The bulge is assumed to be formed by infall of primordial gas described by an exponential law, where $\tau$ is the timescale for the gas accretion.

$$
\frac{d \Sigma_{I}(R, t)}{d t}=A(R) e^{-\frac{t}{\tau}}
$$


where $A(R)$ is a parameter obtained by reproducing the present time total surface mass density of M31's bulge, and $\tau$ is the time scale for gas accretion. The gas present in the bulge is supposed to be well mixed and homogeneous at any time.

We assumed that the star formation rate (SFR) is described by a simple power law (Schmidt 1959; Kennicutt, 1989) regulated by an efficiency $v$ :

$$
\Psi(R, t) \propto v \Sigma_{g}^{k}(R, t)
$$

where $\Sigma_{g}(r, t)$ is the gas surface mass density and $k$ is the slope of the Schmidt law for which we used two different values $k=1.0$ and $k=1.5$. As already demonstrated by other authors (BMOR2007, MB90) a high value for the efficiency in the SFR is needed to reproduce the chemical evolution of the Galactic bulge. In this work we adopt $v=20 G y r^{-1}$ as suggested by MB90. We did not invoke a threshold in the SFR as it was original proposed for self-regulated disks, since there is no evidence that it should take place also in bulges (Elmegreen 1999). However we checked the model using a threshold of $4 M_{\odot} / p c^{-2}$ and no changes were observed in the results.

The instantaneous recycling approximation is relaxed as stellar lifetimes are taken into account following the prescriptions of Kodama (1997). The stellar yields used in this work are those of Woosley \& Weaver (1995) corrected for metallicity dependent oxygen yields as suggested by François et al. (2004). The rate for Type Ia SN is computed according to the work of Matteucci \& Recchi (2001) following the single degenerate scenario for the progenitors of these supernovae.

In order to make the model as simple as possible we invoked a single slope IMF:

$$
\phi(M) \propto M^{-(1+x)}
$$

In our models we are going to test the classical Salpeter IMF $(x=1.35)$ and the MB90 IMF $(x=$ 1.1).

The assumed solar abundances are those derived by Asplund et al. (2009).

The intrinsic parameters for M31 adopted in this work are bulge mass $M_{B M 31}=3.3 \times 10^{10} M_{\odot}$ (although the model proved to be more sensitive to other parameters than the galaxy mass) and bulge radius $R_{B M 31}=4.0 \mathrm{kpc}$ (Geehan et al. 2006).

\section{Results}

In this section we present the predictions for the chemical evolution of the classical bulge of M31. As a first step we computed the model testing different scenarios for the M31 bulge evolution to reproduce the metallicity distribution of dwarf stars by Sarajedini \& Jablonka (2005) and Worthey et al. (2005) (hereafter SJ05 and WEMC05, respectively).

Models parameters can be seen in Table 1, first column is the model name (Salp prefix for those with the Salpeter IMF and MB for those with the MB90 IMF), second column is the value of the exponent in the IMF, third column is the exponent $k$ in the Schimdt law whereas fourth column is for the timescale for the gas infall in Gyr.

\subsection{Metallicity Distribution}

Figure 1 shows the predicted metallicity distribution function (MDF) compared to observational data (filled grey for SJ05 and empty bars for WEMC05). The plots are divided by models, 
Table 1: Models Parameters

\begin{tabular}{cccccccc}
\hline \hline Model & IMF & $k$ & $\begin{array}{c}\tau \\
\text { Gyr }\end{array}$ & Model & IMF & $k$ & $\begin{array}{c}\tau \\
\text { Gyr }\end{array}$ \\
\hline Salp 1-01 & 1.35 & 1.0 & 0.1 & MB 1-01 & 1.1 & 1.0 & 0.1 \\
Salp 1-005 & 1.35 & 1.0 & 0.05 & MB 1-005 & 1.1 & 1.0 & 0.05 \\
Salp 15-01 & 1.35 & 1.5 & 0.1 & MB 15-01 & 1.1 & 1.5 & 0.1 \\
Salp 15-005 & 1.35 & 1.5 & 0.05 & MB 15-005 & 1.1 & 1.5 & 0.05 \\
\hline
\end{tabular}

where the prefix MB or Salp means which IMF was used and the following numbers correspond to the $k$ exponent of the SFR law and the timescale for the infall law, respectively (see table). For each panel, the lines represent different timescales for the infall, solid lines for models with $\tau=0.1 G y r$ while dashed lines are for $\tau=0.05 G y r$.

After the analysis of the MDF plots, one can notice that the models with $k=1$ (left panels) tend to produce more metal rich stars underestimating the number of metal poor objects. Models with higher values of $k$ were tested and provided better results. The right panels of figure 1 shows the same observed metallicity distribution data along with models for which $k=1.5$ was used.
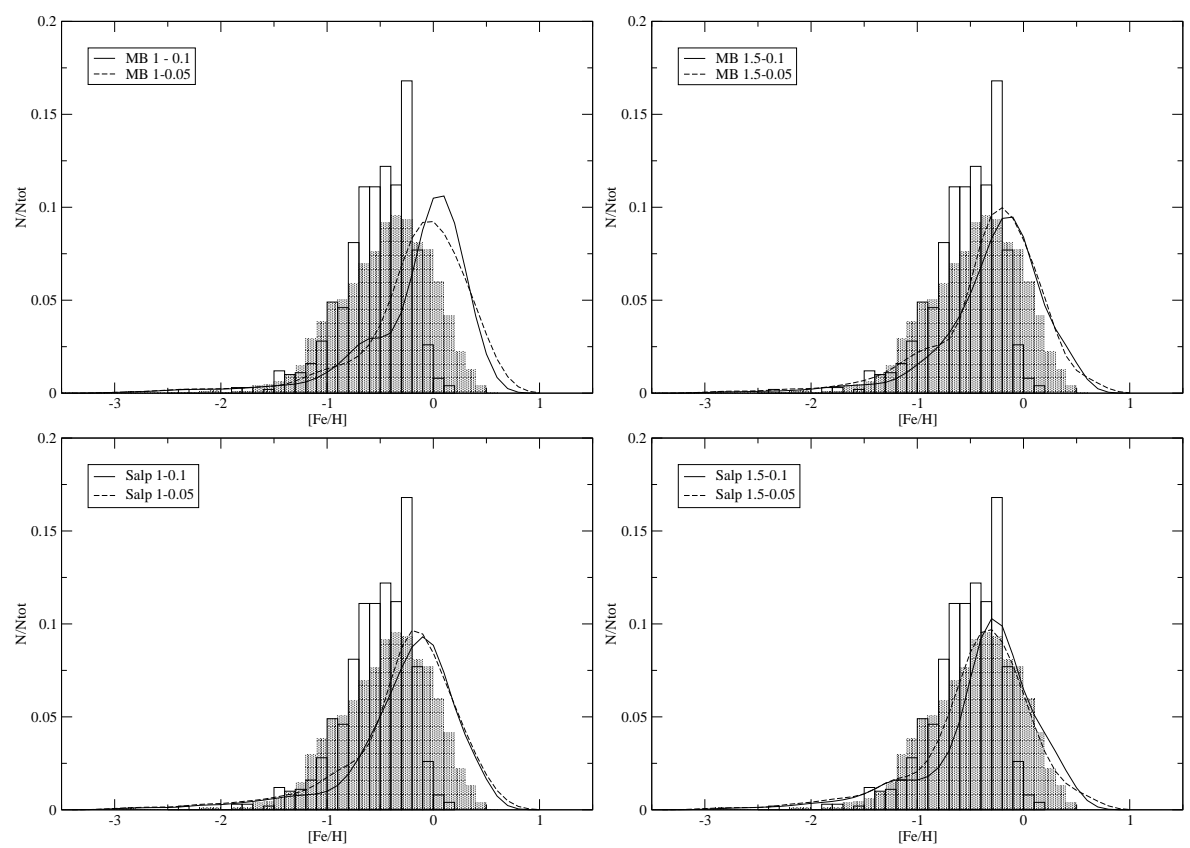

Figure 1: Metallicity distribution for dwarf stars in the bulge of M31. Predicted metallicity distribution function (MDF) compared to observational data (filled grey for SJ05 and empty bars for WEMC05). Upper pannels show the results for MB IMF models while lower pannels show the predictions for the Salpeter's IMF models 


\subsection{Abundance Ratios}

Chemical abundance ratios are a key parameter to understand the chemical evolution of stellar systems. In particular, the $[\alpha / \mathrm{Fe}]$ vs $[\mathrm{Fe} / \mathrm{H}]$ diagram clearly shows the time-delay between the chemical enrichment from SNe type II and SNe type Ia. These ratios can, therefore, be used to constrain chemical evolution models and to provide hints concerning the evolution of the considered system.

To date, there is no alpha-element abundances derived from observational data for the bulge of M31 available in the literature. We now present the predictions for $[\mathrm{O} / \mathrm{Fe}],[\mathrm{Mg} / \mathrm{Fe}],[\mathrm{Si} / \mathrm{Fe}]$, $[\mathrm{Ca} / \mathrm{Fe}],[\mathrm{S} / \mathrm{Fe}],[\mathrm{C} / \mathrm{Fe}]$ and $[\mathrm{N} / \mathrm{Fe}]$ vs $[\mathrm{Fe} / \mathrm{H}]$ for the four models with $k=1.5$ as they were the ones that best fitted the metallicity distribution observed in the bulge of M31 (figure 2). In all panels the solid line represents MB 15-01, the dotted line MB 15-005 while dashed line is for Salp 15-01 and dot-dashed Salp15-005.
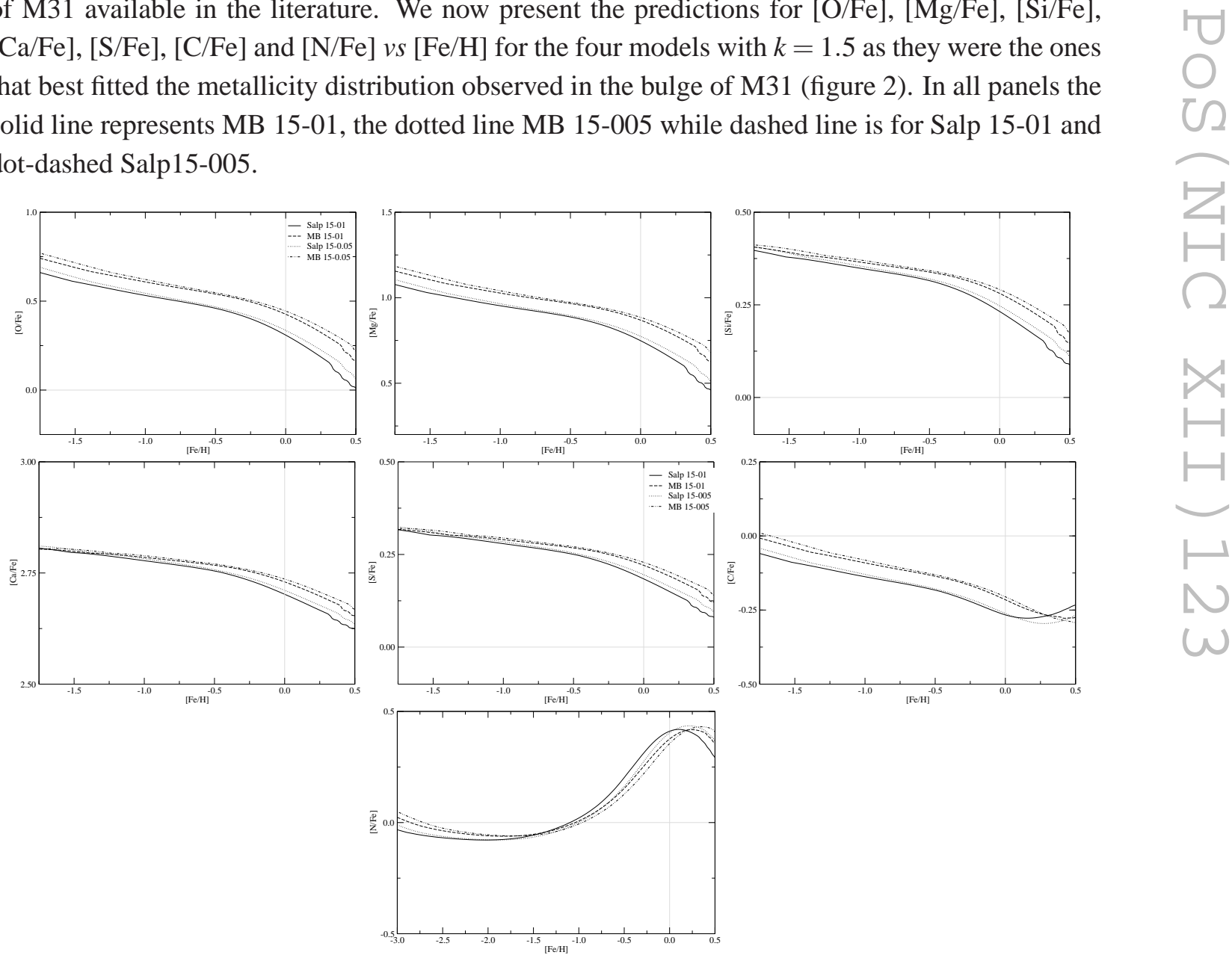

Figure 2: Abundance ratios for the bulge of M31. $\alpha$-elements versus $[\mathrm{Fe} / \mathrm{H}]:[\mathrm{O} / \mathrm{Fe}]$ in the upper left pannel, $[\mathrm{Mg} / \mathrm{Fe}]$ in the upper middle pannel, $[\mathrm{Si} / \mathrm{Fe}]$ in the upper right pannel and $[\mathrm{Ca} / \mathrm{Fe}]$ in the middle left pannel. $[\mathrm{S} / \mathrm{Fe}] \mathrm{x}[\mathrm{Fe} / \mathrm{H}]$ in the middle pannel, $[\mathrm{C} / \mathrm{Fe}] \mathrm{x}[\mathrm{Fe} / \mathrm{H}]$ in the middle right pannel and $[\mathrm{N} / \mathrm{Fe}] \times[\mathrm{Fe} / \mathrm{H}]$ in the lower pannel In all figures solid lines represent the Salp 15-01 model, dashed lines the MB 15-01 model, dotted lines for Salp 15-0.05 whereas dot-dashed lines represent model MB 15-0.05.

\section{Conclusions}

In this work we reproduced the observed MDF of M31 using a generalized chemical evolution 
model for a classical bulge. Our best models to describe the central regions of M31 are the ones with Salpeter IMF and exponent in the SFR law $k=1.5$. The system was formed in a very fast way with high efficiency $\left(v=20 G y r^{-1}\right)$ and small timescales for the infall of gas, reaching values around 0.05 Gyrs.

MB90 and BKM2007 concluded that to reproduce the chemical evolution of the bulge of the Milky Way one has to use a flatter IMF $(x \sim 1.1)$. In the present work we showed that using a classical bulge model with the Salpeter IMF we can reproduce the MDF of the bulge of M31. In fact, many recent works suggest the classification of M31 as a pure classical bulge while new observations of stellar populations in the bulge of the Galaxy support a secular evolution scenario, suggesting that the MW bulge and the central parts of M31 have experienced different star formation histories and evolution. Concerning the $\alpha$-elements distribution we obtain a long plateau and oversolar abundances for the $[\alpha / \mathrm{Fe}]$ vs $[\mathrm{Fe} / \mathrm{H}]$ diagram for $\mathrm{O}, \mathrm{Mg}, \mathrm{Si}, \mathrm{S}$ and $\mathrm{Ca}$ similar to the ones observed for the Milky Way bulge. The abundance predictions for $\alpha$-elements from models with the MB90 IMF present higher values than the models with the Salpeter IMF. Observational measurements of the abundances in the bulge of M31 are needed to better constrain the chemical evolution models. Future observations will be able to verify the validity of our predictions.

We also conclude that the bulge of M31 should have formed very fast relative to other components of this galaxy on a timescale of $0.1 \sim 0.05$ Gyrs, confirming the idea that all classical bulges form on a short timescale. Model Salp15-0.05 is the one that show central tendencies in the MDF more similar to the ones observed by SB05 and WEMC05 and produces less metal rich stars when compared to the same model with a higher timescale (Salp15-0.1).

\section{References}

[Asplund et al.(2009)] Asplund, M., Grevesse, N., Sauval, A. J., \& Scott, P. 2009, ARAA, 47, 481

[Ballero et al.(2007)] Ballero, S. K., Matteucci, F., Origlia, L., \& Rich, R. M. 2007, A\&A, 467, 123

[Ballero et al.(2007)] Ballero, S. K., Kroupa, P., \& Matteucci, F. 2007, A\&A, 467, 117

[Elmegreen(1999)] Elmegreen, B. G. 1999, ApJ, 517, 103

[Geehan et al.(2006)] Geehan, J. J., Fardal, M. A., Babul, A., \& Guhathakurta, P. 2006, MNRAS, 366, 996

[Kennicutt(1989)] Kennicutt, R. C., Jr. 1989, ApJ, 344, 685

[Kodama(1997)] Kodama, T. 1997, Ph.D. Thesis,

[Kormendy \& Kennicutt(2004)] Kormendy, J., \& Kennicutt, R. C., Jr. 2004, ARAA, 42, 603

[Matteucci \& Brocato(1990)] Matteucci, F., \& Brocato, E. 1990, ApJ, 365, 539

[Matteucci(1986)] Matteucci, F. 1986, MNRAS, 221, 911

[McWilliam \& Rich(1994)] McWilliam, A., \& Rich, R. M. 1994, ApJS, 91, 749

[Salpeter(1955)] Salpeter, E. E. 1955, ApJ, 121, 161

[Sarajedini \& Jablonka(2005)] Sarajedini, A., \& Jablonka, P. 2005, AJ, 130, 1627

[Schmidt(1959)] Schmidt, M. 1959, ApJ, 129, 243

[Woosley \& Weaver(1995)] Woosley, S. E., \& Weaver, T. A. 1995, ApJS, 101, 181

[Worthey et al.(2005)] Worthey, G., España, A., MacArthur, L. A., \& Courteau, S. 2005, ApJ, 631, 820 